\title{
INTEGRAL POINTS OF BOUNDED HEIGHT ON COMPACTIFICATIONS OF SEMI-SIMPLE GROUPS
}

\author{
RAMIN TAKLOO-BIGHASH AND YURI TSCHINKEL
}

\begin{abstract}
We study the asymptotic distribution of integral points of bounded height on partial bi-equivariant compactifications of semisimple groups of adjoint type.
\end{abstract}

To Joe Shalika

\section{Contents}

1. Introduction

2. Basic notation

3. Heights and height integrals

4. Infinite-dimensional representations

5. Height zeta function

6. The leading pole

References

\section{INTRODUCTION}

In this paper, we study the asymptotic distribution of $S$-integral points of bounded height on partial bi-equivariant compactifications of semi-simple groups of adjoint type. Our approach combines the spectral techniques developed in [19] in the context of integral points with more recent results in [5], 4], and [6].

Throughout, $\mathrm{G}$ is a split semi-simple group of adjoint type of rank larger than one over a number field $F$ and $X$ the wonderful compactification of G. The boundary $X \backslash G$ is a strict normal crossings divisor $\cup_{\alpha \in \mathcal{A}} D_{\alpha}$ whose set of irreducible components $\mathcal{A}$ is in bijection with the set $\Delta=\Delta(\mathrm{G}, \mathrm{T})$ of simple roots of $\mathrm{G}$, with respect to a fixed maximal split torus $\mathrm{T}$ of $\mathrm{G}$. Each $A \subseteq \Delta$ corresponds to the boundary stratum $D_{A}:=\cap_{\alpha \in A} D_{\alpha}$. A rational character $\lambda \in \mathfrak{X}^{*}(\mathrm{~T})$ gives rise to a line bundle $L_{\lambda}$ on $X$, the classes of such line bundles span the Picard group $\operatorname{Pic}(X)$ of $X$, after tensoring with $\mathbb{Q}$ (see [19, Section 5]). In [19] we defined a height pairing

$$
H: \mathfrak{X}^{*}(\mathrm{~T})_{\mathbb{C}} \times \mathrm{G}(\mathbb{A}) \rightarrow \mathbb{C}
$$

Date: November 13, 2018. 
whose restriction to $\lambda \times \mathrm{G}(F)$ is a a standard Weil height function on $X$ with respect to $L_{\lambda}$. The height function is left- and right invariant with respect to the action of a maximal compact subgroup $\mathrm{K}=\prod_{v} \mathrm{~K}_{v} \subset \mathrm{G}(\mathbb{A})$ of the adelic points of $\mathrm{G}$. We established an asymptotic formula for the number

$$
N(B, \lambda):=\#\{\gamma \in \mathrm{G}(F) \mid H(\lambda, \gamma) \leq B\}, \quad B \rightarrow \infty,
$$

for all $\lambda$ corresponding to big line bundles on $X$, i.e., all classes in the interior of the effective cone in $\operatorname{Pic}(X)_{\mathbb{R}}$.

From now on we fix a divisor $D \subset X \backslash \mathrm{G}$ and let $\mathcal{A}_{D}$ be the set of $\alpha$ such that $D=\cup_{\alpha \in \mathcal{A}_{D}} D_{\alpha}$. Let $S$ be a finite set of places of $F$ containing the archimedean places. We introduce the set-theoretic characteristic function $\boldsymbol{\delta}=\boldsymbol{\delta}_{D, S}$ of the set of $(D, S)$-integral points on $X$ as follows: For $v \notin S$, let

$$
\boldsymbol{\delta}_{v}: \mathrm{G}\left(F_{v}\right) \rightarrow\{0,1\}
$$

be given by

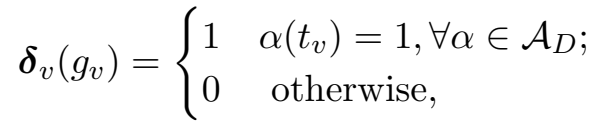

where

$$
g_{v}=k_{v} t_{v} k_{v}^{\prime}, \quad \text { with } \quad t_{v} \in \mathrm{T}\left(F_{v}\right), k_{v}, k_{v}^{\prime} \in \mathrm{K}_{v} .
$$

For $v \in S$, we put $\boldsymbol{\delta}_{v} \equiv 1$ and write

$$
\boldsymbol{\delta}:=\prod_{v} \boldsymbol{\delta}_{v}
$$

A point $\gamma \in \mathrm{G}(F) \subset \mathrm{G}(\mathbb{A})$ is called $(D, S)$-integral if $\boldsymbol{\delta}(\gamma)$ is equal to 1 . This definition agrees with the usual definition of $S$-integral points on a model of $X \backslash D$ over the integers. The corresponding counting functions are given by

$$
N_{S, D}(B, \lambda)=\#\{(D, S) \text { - integral } \quad \gamma \in \mathrm{G}(F) \mid H(\lambda, \gamma) \leq B\} .
$$

Our main result is Theorem 6.4 which shows that

$$
N_{S, D}(\lambda, B)=c \cdot B^{a} \log (B)^{b-1}(1+o(1)), \quad B \rightarrow \infty,
$$

with an explicit bound on the error term. The constants $a, b$, and $c$ involve arithmetic, geometric, and combinatorial information about the data $X, D$, $\lambda$, and $S$. Our Theorem 6.4 can be considered as an interpolation between results on rational points in [19] and [11] and on integral points on G in [13, 12. It is consistent with a generalization of the conjecture of BatyrevManin and Peyre to the framework of integral points proposed in [5], [4], and [6].

As in [19] our method is based on the spectral analysis of the height zeta function, modified to count $(D, S)$-integral points. We prove that the main poles of the height zeta function arise from the contribution of unramified one-dimensional automorphic representations of $G(\mathbb{A})$, the set of which is denoted by $\mathcal{X}(\mathrm{G})$. 
In rank one, i.e., for $\mathrm{G}=\operatorname{PGL}(2)$, the spectral theory and the height integrals have been completely worked out in [18]. The wonderful compactification of $\mathrm{PGL}(2)$ is the projective space $\mathbb{P}^{3}$, with a unique irreducible boundary component which is a smooth split quadric, and the distribution of $(D, S)$-integral points is an interesting classical problem.

The study of $S$-integral points on algebraic varieties has a rich history, going back at least as far as Siegel's work on algebraic curves. More recently the distribution of integral points on affine homogeneous varieties has been studied in several papers, e.g., in [9, 3, 15], as well as in [10] where ergodictheoretic methods are employed. Ergodic-theoretic methods are also used in [2] to treat fairly general homogeneous varieties. Our method, in technique and spirit, is quite close to [4, 6]. An introduction to integral points for pairs $(X, D)$ can be found [14]. For background concerning the geometry of wonderful compactifications, see [7]. Arithmetic properties of these varieties, in connection with counting of rational points of bounded height, are discussed in [19].

The paper is organized as follows. In Section 3 we review the theory of height functions from the group-theoretic perspective and study local and global height integrals. Section 4 is devoted to the regularization of adelic height integrals. Section 5 contains our main theorem and its proof.

Acknowledgments. We are grateful to Wee-Teck Gan for helpful remarks. The first author was partially supported by NSF grant DMS-0701753. The second author was partially supported by NSF grants DMS-0739380 and 0901777.

\section{BASIC NOTATION}

For $X$ an algebraic variety over a field $F$ we write $X(F)$ for the set of $F$-rational points of $X$. We denote by $\operatorname{Pic}(X)$ the Picard group of $X$ and by $\Lambda_{\mathrm{eff}}(X) \subset \operatorname{Pic}(X)_{\mathbb{R}}$ the (closed) cone of effective divisors on $X$. We often identify line bundles, divisors and their classes in $\operatorname{Pic}(X)$.

From now on, we let $F$ be a number field, $\operatorname{Val}(F)$ the set its places, and $S_{\infty}$ the set of archimedean places. For $v \in \operatorname{Val}(F)$, let $F_{v}$ be the completion of $F$ with respect to $v, \varpi_{v}$ a uniformizer at $v$, and $q=q_{v}$ the order of the residue field. For any finite set of places $S$ (containing $S_{\infty}$ ) we denote by $\mathfrak{o}_{S}$ the ring of $S$-integers of $F$. We denote by $\mathbb{A}$ the ring of adeles, by $\mathbb{A}_{S}=\prod_{v \notin S}^{\prime} F_{v}$ and by $\mathbb{A}_{f}=\mathbb{A}_{S_{\infty}}$. All Haar measures $\mathrm{d} g_{v}$ will be normalized as in [1]; we put $\mathrm{d} g_{S}=\prod_{v \notin S} \mathrm{~d} g_{v}$ and $\mathrm{d} g=\prod_{v} \mathrm{~d} g_{v}$.

We fix a connected split semi-simple group $G$ of adjoint type over $F$ or rank $r \geq 2$. Let $\mathrm{T}$ be a maximal split torus of $\mathrm{G}$ and $\mathrm{B} \subset \mathrm{G}$ a Borel subgroup containing $\mathrm{T}$. We also let $\Delta=\Delta(\mathrm{G}, T)$ be the set of simple roots for $(\mathrm{G}, \mathrm{T})$, 
with respect to $\mathrm{B}$, and $\rho$ half the sum of positive roots. We have

$$
2 \rho=\sum_{\alpha \in \Delta} \kappa_{\alpha} \alpha
$$

for some constants $\kappa_{\alpha} \geq 1$. We set

$$
\hat{F}_{v}:= \begin{cases}\{x \in \mathbb{R} \mid x \geq 1\} & \text { if } v \mid \infty, \\ \left\{\varpi_{v}^{-n} \mid n \in \mathbb{N}\right\} & \text { if } v \nmid \infty,\end{cases}
$$

and

$$
\mathrm{T}\left(F_{v}\right)^{+}=\left\{a \in \mathrm{T}\left(F_{v}\right) \mid \alpha(a) \in \hat{F}_{v} \text { for each } \alpha \in \Phi^{+}\right\} .
$$

We have the following Cartan decomposition:

$$
\mathrm{G}\left(F_{v}\right)=\mathrm{K}_{v} \mathrm{~T}\left(F_{v}\right)^{+} \mathrm{K}_{v}
$$

\section{Heights And height Integrals}

We identify the set $\mathcal{A}=\left\{D_{\alpha}\right\}$ of boundary components with $\Delta=\Delta(\mathrm{G}, \mathrm{T})$. The height pairing

$$
\operatorname{Pic}(X)_{\mathbb{C}} \times \mathrm{G}(\mathbb{A}) \rightarrow \mathbb{C}
$$

is given by

$$
H(\mathbf{s}, g)=\prod_{v} H_{v}\left(\mathbf{s}, g_{v}\right), \quad \text { where } \quad H_{v}\left(\mathbf{s}, g_{v}\right)=\prod_{\alpha \in \mathcal{A}}\left|\alpha\left(t_{v}\right)\right|_{v}^{s_{\alpha}},
$$

for $\mathbf{s}=\left(s_{\alpha}\right)_{\alpha \in \mathcal{A}}$, and

$$
g=\left(g_{v}\right)_{v} \in \mathrm{G}(\mathbb{A}) \quad \text { with } g_{v}=k_{v} t_{v} k_{v}^{\prime}, \quad k_{v}, k_{v}^{\prime} \in \mathrm{K}_{v}, \quad t_{v} \in \mathrm{T}\left(F_{v}\right)^{+},
$$

(see Section 6 of [19]). We will also use

$$
H_{S}(\mathbf{s}, g):=\prod_{v \notin S} H_{v}\left(\mathbf{s}, g_{v}\right) .
$$

We have

$$
-K_{X}=\sum_{\alpha \in \mathcal{A}}\left(\kappa_{\alpha}+1\right) D_{\alpha}
$$

see [19, Proposition 5.2]. For $\epsilon \in \mathbb{R}$, let

$$
\mathcal{T}_{\epsilon}=\left\{\mathbf{s} \mid \Re\left(s_{\alpha}\right)>\kappa_{\alpha}+1+\epsilon, \quad \text { for all } \alpha\right\}
$$

and

$$
\mathcal{T}_{\epsilon}^{D}=\left\{\mathbf{s} \mid \Re\left(s_{\alpha}\right)>\kappa_{\alpha}+1+\epsilon, \quad \text { for all } \alpha \notin \mathcal{A}_{D}\right\}
$$

The following theorem has been proved in [19, Sections 6.6 and 6.7].

Theorem 3.1. Let

$$
\mathcal{J}_{v}(\mathbf{s}):=\int_{\mathrm{G}\left(F_{v}\right)} H_{v}\left(\mathbf{s}, g_{v}\right)^{-1} \mathrm{~d} g_{v} .
$$

(1) For $v \notin S_{\infty}$ the integral $\mathcal{J}_{v}(\mathbf{s})$ is a holomorphic function for $\mathbf{s} \in \mathcal{T}_{-1}$. 
(2) For $v \in S_{\infty}$ and $\partial$ in the universal enveloping algebra the integral

$$
\mathcal{J}_{v, \partial}(\mathbf{s}):=\int_{\mathrm{G}\left(F_{v}\right)} \partial\left(H_{v}\left(\mathbf{s}, g_{v}\right)^{-1}\right) \mathrm{d} g_{v}
$$

is holomorphic for $\mathbf{s} \in \mathcal{T}_{-1}$.

Moreover, for all $\epsilon>0$ and $\partial$, there exist constants $C_{v}(\epsilon)$ and $C_{v}(\partial, \epsilon)$ such that

$$
\left|\mathcal{J}_{v}(\mathbf{s})\right| \leq C_{v}(\epsilon) \quad \text { and } \quad\left|\mathcal{J}_{v, \partial}(\mathbf{s})\right| \leq C_{v}(\partial, \epsilon), \quad \text { for all } \quad \mathbf{s} \in \mathcal{T}_{-1+\epsilon} .
$$

The following result generalizes the computations in [19, Sections 6 and $7]$ to the $(D, S)$-integral context.

Theorem 3.2. Let $\chi=\otimes_{v}^{\prime} \chi_{v}$ be a one-dimensional unramified automorphic representation of $\mathrm{G}(\mathbb{A})$, and $S$ and $D$ as in Section 1 . For each $v \in S$ here exists a function $f_{v}$, holomorphic and uniformly bounded in $\mathcal{T}_{-1-\delta}$, for some $\delta>0$, such that

$$
\int_{\mathrm{G}\left(F_{v}\right)} H_{v}\left(\mathbf{s}, g_{v}\right)^{-1} \chi_{v} \mathrm{~d} g_{v}=\prod_{\alpha \in \mathcal{A}} L_{v}\left(s_{\alpha}-\kappa_{\alpha}, \chi_{v} \circ \check{\alpha}\right) \cdot f_{v}(\mathbf{s}),
$$

where $L_{v}$ is the local factor of the Hecke L-function.

Moreover, there exists a function $f_{S, \chi}$, which depends only on $\left(s_{\alpha}\right)_{\alpha \notin \mathcal{A}_{D}}$, is holomorphic in $\mathcal{T}_{-1 / 2}^{D}$, uniformly bounded in $\mathcal{T}_{-1 / 2+\epsilon}^{D}$, for any $\epsilon>0$, and such that

$\mathcal{J}_{S, D}(\mathbf{s}, \chi):=\int_{\mathrm{G}\left(\mathbb{A}_{S}\right)} \boldsymbol{\delta}_{S}(g) H_{S}(\mathbf{s}, g)^{-1} \chi(g) \mathrm{d} g=\prod_{\alpha \notin \mathcal{A}_{D}} L\left(s_{\alpha}-\kappa_{\alpha}, \chi \circ \check{\alpha}\right) \cdot f_{S, \chi}(\mathbf{s})$.

Proof. The first claim follows from [5, Proposition 4.1.2], see also [19, Theorem 6.9].

To prove the second claim, let

$$
\mathcal{J}_{S, D}(\mathbf{s}, \chi)=\prod_{v \notin S} \mathcal{J}_{v}\left(\mathbf{s}, \chi_{v}\right)
$$

where

$$
\mathcal{J}_{v}\left(\mathbf{s}, \chi_{v}\right)=\int_{\mathrm{G}\left(F_{v}\right)} \boldsymbol{\delta}_{v}\left(g_{v}\right) H_{v}\left(\mathbf{s}, g_{v}\right)^{-1} \chi_{v}\left(g_{v}\right) \mathrm{d} g_{v} .
$$

Since $G$ is of adjoint type, the collection of elements $\left\{\check{\alpha}\left(\varpi_{v}\right)\right\}_{\alpha \in \Delta}$ forms a basis for the semi-group $\mathrm{T}_{v}\left(F_{v}\right)^{+}$. For $\mathbf{a}=\left(a_{\alpha}\right)_{\alpha \in \Delta}$ we set

$$
t_{v}(\mathbf{a})=\prod_{\alpha \in \mathcal{A}} \check{\alpha}\left(\varpi_{v}\right)^{a_{\alpha}} \in \mathrm{T}\left(F_{v}\right)^{+} .
$$

Using the bi-K-invariance we may write the local integrals as

$$
\mathcal{J}_{v}\left(\mathbf{s}, \chi_{v}\right)=\sum_{\mathbf{a} \in \mathbb{N}^{r}} \boldsymbol{\delta}_{v}\left(t_{v}(\mathbf{a})\right) q_{v}^{-\langle\mathbf{s}, \mathbf{a}\rangle} \chi_{v}\left(t_{v}(\mathbf{a})\right) \operatorname{vol}\left(\mathrm{K}_{v} t_{v}(\mathbf{a}) \mathrm{K}_{v}\right),
$$


where

$$
\langle\mathbf{s}, \mathbf{a}\rangle=\sum_{\alpha \in \mathcal{A}} s_{\alpha} a_{\alpha}
$$

By [19, Lemma 6.11], there exists a constant $C$, independent of $v$, such that for all $t_{v}(\mathbf{a}) \in \mathrm{T}\left(F_{v}\right)^{+}$, one has

$$
\operatorname{vol}\left(\mathrm{K}_{v} t_{v}(\mathbf{a}) \mathrm{K}_{v}\right) \leq \delta_{\mathrm{B}}\left(t_{v}(\mathbf{a})\right)\left(1+\frac{C}{q_{v}}\right)
$$

where

$$
\delta_{\mathrm{B}}\left(t_{v}(\mathbf{a})\right):=\left|\rho\left(t_{v}(\mathbf{a})\right)\right|_{v}^{2}=q_{v}^{\langle 2 \rho, \mathbf{a}\rangle}
$$

We may rewrite Equation (6) as

$$
\mathcal{J}_{v}\left(\mathbf{s}, \chi_{v}\right)=\sum_{\mathbf{a} \in \mathbb{N}^{r}} \boldsymbol{\delta}_{v}\left(t_{v}(\mathbf{a})\right) q_{v}^{-\langle\mathbf{s}-2 \rho, \mathbf{a}\rangle} \chi_{v}\left(t_{v}(\mathbf{a})\right)+b_{v}(\mathbf{s})
$$

where

$$
b_{v}(\mathbf{s})=\sum_{\mathbf{a} \in \mathbb{N}^{r}} \boldsymbol{\delta}_{v}\left(t_{v}(\mathbf{a})\right) q_{v}^{-\langle\mathbf{s}, \mathbf{a}\rangle} \chi_{v}\left(t_{v}(\mathbf{a})\right)\left(\operatorname{vol}\left(\mathrm{K}_{v} t_{v}(\mathbf{a}) \mathrm{K}_{v}\right)-\delta_{\mathrm{B}}\left(t_{v}(\mathbf{a})\right)\right)
$$

Observe that

(8)

$$
\begin{aligned}
\sum_{\mathbf{a} \in \mathbb{N}^{\mathbf{r}}} \boldsymbol{\delta}_{v}\left(t_{v}(\mathbf{a})\right) q_{v}^{-\langle\mathbf{s}-\mathbf{2} \rho, \mathbf{a}\rangle} \chi_{v}\left(t_{v}(\mathbf{a})\right) & =\prod_{\alpha \notin \mathcal{A}_{D}}\left(\sum_{a_{\alpha}=0}^{\infty} \chi_{v}\left(\check{\alpha}\left(\varpi_{v}\right)\right)^{a_{\alpha}} q_{v}^{-\left(s_{\alpha}-\kappa_{\alpha}\right) a_{\alpha}}\right) \\
& =\prod_{\alpha \notin \mathcal{A}_{D}} \frac{1}{1-\chi_{v}\left(\check{\alpha}\left(\varpi_{v}\right)\right) q_{v}^{-\left(s_{\alpha}-\kappa_{\alpha}\right)}} .
\end{aligned}
$$

The corresponding Euler product, over $v \notin S$, is a product of partial $L$ functions, as in the statement of the theorem.

Let $\sigma=\left(\Re\left(s_{\alpha}\right)\right)_{\alpha}$. In the definition $b_{v}$, we may assume $\mathbf{a} \neq \underline{0}$. Since for each $v \notin S$,

$$
\{\mathbf{a} \mid \mathbf{a} \neq \underline{0}\}=\bigcup_{\alpha \in \mathcal{A}}\left\{\mathbf{a} ; a_{\alpha} \neq 0\right\},
$$


we have

$$
\begin{aligned}
\sum_{v \notin S}\left|b_{v}(\mathbf{s})\right| & \leq \sum_{v \notin S} \sum_{\alpha} \sum_{a_{\alpha} \neq 0} \boldsymbol{\delta}_{v}\left(t_{v}(\mathbf{a})\right) q_{v}^{-\langle\sigma, \mathbf{a}\rangle}\left|\left(\operatorname{vol}\left(\mathrm{K}_{v} t_{v}(\mathbf{a}) \mathrm{K}_{v}\right)-\delta_{\mathrm{B}}\left(t_{v}(\mathbf{a})\right)\right)\right| \\
& \ll \sum_{v \notin S} q_{v}^{-1} \sum_{\alpha \notin \mathcal{A}_{D}} \sum_{a_{\alpha} \neq 0} q_{v}^{-\langle\sigma, \mathbf{a}\rangle} \delta_{\mathrm{B}}\left(t_{v}(\mathbf{a})\right) \\
& =\sum_{v \notin S} q_{v}^{-1} \sum_{\alpha \notin \mathcal{A}_{D}}\left(\sum_{a_{\alpha}=1}^{\infty} q_{v}^{-\left(\sigma_{\alpha}-\kappa_{\alpha}\right) a_{\alpha}}\right) \prod_{\substack{\beta \neq \alpha \\
\beta \notin \mathcal{A}_{D}}}\left(\sum_{a_{\beta}=0}^{\infty} q_{v}^{-\left(\sigma_{\beta}-\kappa_{\beta}\right) a_{\beta}}\right) \\
& =\sum_{v \notin S} q_{v}^{-1} \sum_{\alpha \notin \mathcal{A}_{D}} \frac{q_{v}^{-\left(\sigma_{\alpha}-\kappa_{\alpha}\right)}}{\prod_{\beta \notin \mathcal{A}_{D}}\left(1-q_{v}^{-\left(\sigma_{\beta}-\kappa_{\beta}\right)}\right)} \\
& \ll \sum_{\alpha \notin \mathcal{A}_{D}} \sum_{v \notin S} q_{v}^{-\frac{3}{2}}<\infty .
\end{aligned}
$$

Note that for $\mathbf{s} \in \mathcal{T}_{-1 / 2+\epsilon}^{D}$ the estimates are uniform and the corresponding function $f_{S, \chi}$ is holomorphic in $\mathcal{T}_{-1 / 2+\epsilon}^{D}$.

\section{INFINITE-DIMENSIONAL REPRESENTATIONS}

Let $\pi=\otimes_{v}^{\prime} \pi_{v}$ be an infinite-dimensional automorphic representation of $\mathrm{G}(\mathbb{A})$ and $\varphi_{\pi_{v}}$ the normalized spherical function associated to $\pi_{v}$. Since $\mathrm{G}$ is split, the representation $\pi_{v}$ is infinite-dimensional, for all $v$ (see [19, Proposition 4.4]). We need uniform upper bounds for $\varphi_{\pi_{v}}$. We will use the following special case of a result of Hee Oh [16, Theorem 1.1].

Proposition 4.1. Assume that the rank $r$ of $\mathrm{G}$ is at least two. Then for each $\alpha \in \Delta$ and $g_{v}=k_{v} t_{v} k_{v}^{\prime}$ we have

$$
\left|\varphi_{v}\left(g_{v}\right)\right| \leq\left|\alpha\left(t_{v}\right)\right|^{-1 / 2}\left(\frac{\left(\log _{q_{v}}\left|\alpha\left(t_{v}\right)\right|\right)\left(q_{v}-1\right)+\left(q_{v}+1\right)}{q_{v}+1}\right) .
$$

Corollary 4.2. Assume that the rank $r$ of $\mathrm{G}$ is at least two. Then for each $\alpha \in \Delta$ we have

$$
\left|\varphi_{\pi_{v}}\left(\check{\alpha}\left(\varpi_{v}\right)\right)\right|<2 q_{v}^{-\frac{1}{2}} .
$$

Moreover, for all $\epsilon>0$ there is a constant $C_{\epsilon}>0$ such that for $g_{v}=k_{v} t_{v} k_{v}^{\prime}$ we have

$$
\left|\varphi_{\pi_{v}}\left(g_{v}\right)\right| \leq C_{\epsilon} \prod_{\alpha \in \Delta}\left|\alpha\left(t_{v}\right)\right|^{-\frac{1}{2 r}+\epsilon} .
$$

Proof. Equation (9) implies that for all $\epsilon>0$,

$$
\left|\varphi_{\pi_{v}}\left(g_{v}\right)\right| \leq C_{\epsilon}(\alpha)\left|\alpha\left(t_{v}\right)\right|^{-\frac{1}{2}+r \epsilon},
$$

for some constant $C_{\epsilon}(\alpha)$. Multiplying these inequalities over all $\alpha \in \Delta$ and taking $r$ th root gives the result. 
Set

$$
\mathcal{J}_{v}\left(\mathbf{s}, \pi_{v}\right)=\int_{\mathrm{G}\left(F_{v}\right)} \boldsymbol{\delta}_{v}\left(g_{v}\right) H_{v}\left(\mathbf{s}, g_{v}\right)^{-1} \varphi_{\pi_{v}}\left(g_{v}\right) \mathrm{d} g_{v} .
$$

Note that for $v \notin S$ this integral depends only on $\left(s_{\alpha}\right)_{\alpha \notin \mathcal{A}_{D}}$.

Theorem 4.3. The infinite product

$$
\mathcal{J}_{S, D}(\mathbf{s}, \pi):=\prod_{v \notin S} \mathcal{J}_{v}\left(\mathbf{s}, \pi_{v}\right)
$$

is holomorphic for $\mathbf{s} \in \mathcal{T}_{-1 / 2 r}^{D}$. Moreover, for all $\epsilon>0$ and all compacts $K \subset \mathcal{T}_{-1 / 2 r+\epsilon}^{D}$ there exists a constant $C(\epsilon, K)$, independent of $\pi$, such that

$$
\left|\mathcal{J}_{S, D}(\mathbf{s}, \pi)\right| \leq C(\epsilon, K) \text {. }
$$

for all $\mathbf{s} \in K$.

Proof. Using bi-K-invariance, as in the proof of Theorem 3.2, we obtain

$$
\mathcal{J}_{v}\left(\mathbf{s}, \pi_{v}\right)=\sum_{\mathbf{a} \in \mathbb{N}^{r}} \boldsymbol{\delta}_{v}\left(t_{v}(\mathbf{a})\right) q_{v}^{-\langle\mathbf{s}, \mathbf{a}\rangle} \varphi_{\pi_{v}}\left(t_{v}(\mathbf{a})\right) \operatorname{vol}\left(\mathrm{K}_{v} t_{v}(\mathbf{a}) \mathrm{K}_{v}\right) .
$$

Again, $\mathcal{J}_{v}\left(\mathbf{s}, \pi_{v}\right)$ only depends on $s_{\alpha}$ with $\alpha \notin \mathcal{A}_{D}$. Using the estimates from Corollary 4.2 and Equation (7) we conclude that to establish the convergence of the Euler product it suffices to bound

$$
\sum_{v \notin S} \sum_{\left(a_{\alpha}\right)} q_{v}^{-\left(\sum_{\alpha \notin \mathcal{A}_{D}}\left(s_{\alpha}-\kappa_{\alpha}+1 / 2 r-\epsilon\right) a_{\alpha}\right)},
$$

where the inner sum is over nonzero vectors $\left(a_{\alpha}\right) \in \mathbb{N} \mathcal{A} \backslash \mathcal{A}_{D}$.

Now we consider integrals of the form

$$
\mathcal{J}_{v}\left(\mathbf{s}, \varphi_{\pi_{v}}\right):=\int_{G\left(F_{v}\right)} H_{v}\left(\mathbf{s}, g_{v}\right)^{-1} \varphi_{\pi_{v}}\left(g_{v}\right) \mathrm{d} g_{v},
$$

for $v \in S$.

Theorem 4.4. (1) For all $v \notin S_{\infty}$ the integral $\mathcal{J}_{v}\left(\mathbf{s}, \varphi_{v}\right)$ is holomorphic for $\mathbf{s} \in \mathcal{T}_{-1-1 / 2 r}$.

(2) For $v \in S_{\infty}$ and $\partial$ in the universal enveloping algebra the integral

$$
\mathcal{J}_{v, \partial}\left(\mathbf{s}, \varphi_{\pi_{v}}\right):=\int_{\mathbf{G}\left(F_{v}\right)} \partial\left(H_{v}\left(\mathbf{s}, g_{v}\right)^{-1}\right) \varphi_{\pi_{v}}(g) \mathrm{d} g_{v}
$$

is holomorphic for $\mathbf{s} \in \mathcal{T}_{-1-1 / 2 r}$. 
Proof. We verify the non-archimedean statement; the other argument is similar. Let $\underline{\sigma}$ be the vector consisting of the real parts of the components of $\mathbf{s}$. Fix $\epsilon>0$. The local height integral is majorized by

$$
\begin{aligned}
& \sum_{t \in \mathrm{T}\left(F_{v}\right)^{+}} H_{v}(\underline{\sigma}, t)^{-1}\left|\varphi_{v}(t)\right| \delta_{B}(t) \\
\ll & \prod_{\alpha \in \mathcal{A}} \sum_{l=0}^{\infty} H_{v}\left(\underline{\sigma}, \check{\alpha}\left(\varpi_{v}^{l}\right)\right)^{-1} q_{v}^{-(1 / 2 r-\epsilon) l} \delta_{B}\left(\check{\alpha}\left(\varpi_{v}^{l}\right)\right) \\
= & \prod_{\alpha \in \mathcal{A}} \sum_{l=0}^{\infty} q_{v}^{-\left(\sigma_{\alpha}-\kappa_{\alpha}+1 / 2 r-\epsilon\right) l} .
\end{aligned}
$$

The result is now immediate.

Corollary 4.5. In the non-archimedean situation, for each $\epsilon>0$ there is a constant $C_{v}(\epsilon)$, such that $\left|\mathcal{J}_{v}\left(\mathbf{s}, \varphi_{\pi_{v}}\right)\right| \leq C_{v}(\epsilon)$ for all $\mathbf{s} \in \mathcal{T}_{-1-1 / 2 r+\epsilon}$. In the archimedean situation, for all $\epsilon>0$ and all $\partial$ as above, there is a constant $C_{v}(\partial, \epsilon)$ such that $\left|\mathcal{J}_{v, \partial}\left(\mathbf{s}, \varphi_{\pi_{v}}\right)\right| \leq C_{v}(\partial, \epsilon)$ for all $\mathbf{s} \in \mathcal{T}_{-1-1 / 2 r+\epsilon}$.

Corollary 4.6. Let $\varphi$ be an automorphic form in the space of an automorphic representation $\pi$ which is right invariant under the maximal compact subgroup K. Set for $\mathbf{s} \in \mathcal{T}_{\gg 0}$

$$
\mathcal{J}_{S, D}(\mathbf{s}, \varphi):=\int_{\mathbf{G}(\mathbb{A})} \boldsymbol{\delta}_{S, D}(g) H(\mathbf{s}, g)^{-1} \varphi(g) d g .
$$

Then $\mathcal{J}_{S, D}(s, \varphi)$ has an analytic continuation to a function which is holomorphic on $\mathcal{T}_{-1 / 2}^{D} \cap \mathcal{T}_{-1-1 / 2 r}$. Let $\boldsymbol{\Delta}$ be the Laplacian as in the proof of [1. Lemma 4.1], and suppose $\varphi$ is an eigenfunction for $\boldsymbol{\Delta}$. Define $\boldsymbol{\Lambda}(\phi)$ by $\boldsymbol{\Delta} \cdot \varphi=\boldsymbol{\Lambda}(\varphi) \cdot \phi$. Then for each integer $k>0$, all $\epsilon>0$, and every compact subset $K \subset \mathcal{T}_{-1 / 2 r+\epsilon}^{D} \cap \mathcal{T}_{-1-1 / 2 r+\epsilon}$, there exists a constant $C=C(\epsilon, K, k)$, independent of $\varphi$, such that

$$
\left|\mathcal{J}_{S, D}(\mathbf{s}, \varphi)\right| \leq C \boldsymbol{\Lambda}(\varphi)^{-k}|\varphi(e)|
$$

for all $\mathbf{s} \in K$.

\section{Height ZETA FUnCTION}

The main tool in the study of distribution properties of $(S, D)$-integral points is the height zeta function, defined by

$$
\mathcal{Z}_{S, D}(\mathbf{s}, g):=\sum_{\gamma \in \mathrm{G}(F)} \boldsymbol{\delta}_{S, D}(\gamma g) H(\mathbf{s}, \gamma g)^{-1}
$$

Proposition 5.1. The series defining $\mathcal{Z}_{S, D}(\mathbf{s}, g)$ converges absolutely to a holomorphic function for $\mathbf{s} \in \mathcal{T}_{\gg 0}$. In its region of convergence

$$
\mathcal{Z}_{S, D}(\mathbf{s}, g) \in \mathrm{C}^{\infty}(\mathrm{G}(F) \backslash \mathrm{G}(\mathbb{A})) .
$$


and all of its group derivatives are in $\mathrm{L}^{2}$. Moreover, in this domain, we have a spectral expansion

$$
\sum_{\chi \in \mathfrak{X}, \mathrm{P}} \frac{1}{n(\mathrm{~A})} \int_{\Pi(\mathrm{M})} \int_{\mathrm{G}(\mathbb{A})} \boldsymbol{\delta}_{S, D}\left(g^{\prime}\right) H\left(\mathbf{s}, g^{\prime}\right)^{-1}\left(\sum_{\phi \in \mathcal{B}_{\mathrm{P}}(\pi)_{\chi}} E(g, \phi) \overline{E\left(g^{\prime}, \phi\right)}\right) \mathrm{d} g^{\prime} \mathrm{d} \pi,
$$

in the notations of [19, Section 3].

Proof. Identical to the proof of [19, Proposition 8.2]; it suffices to observe that $\mathcal{Z}_{S, D}$ is a subsum of the series defining the height zeta function for rational points considered in [19].

Let $\mathcal{X}=\mathcal{X}(\mathrm{G})$ be the set of unramified automorphic characters of $\mathrm{G}$, i.e., continuous homomorphisms $\mathrm{G}(\mathbb{A}) \rightarrow \mathbb{S}^{1}$, invariant under $\mathrm{G}(F)$ and $\mathrm{K}$ on both sides. We specialize to $g=e$, the identity in $\mathrm{G}(\mathbb{A})$, and obtain

$$
\mathcal{Z}_{S, D}(\mathbf{s}):=\mathcal{Z}_{S, D}(\mathbf{s}, e)=\sum_{\chi \in \mathcal{X}(\mathrm{G})} \int_{\mathrm{G}(\mathbb{A})} \boldsymbol{\delta}_{S, D}(g) H(\mathbf{s}, g)^{-1} \chi(g) \mathrm{d} g+S^{b}(\mathbf{s}),
$$

and $S^{b}(\mathbf{s})$ is the subsum in Equation 14 corresponding to infinite dimensional representations (restricted to $g=e$ ).

We use this expansion to determine the analytic behavior of the height zeta function. The innermost sum in the definition of $S^{b}(\mathbf{s})$ is uniformly convergent for $g^{\prime}$ in compact sets, see the first half of the proof of Lemma 4.4 of [1]. Therefore, we may interchange the innermost summation with the integral over $\mathrm{G}(\mathbb{A})$ and find that $S^{b}(\mathbf{s})$ equals

$$
\sum_{\chi \in \mathfrak{X}, \mathrm{P}}^{b} \frac{1}{n(\mathrm{~A})} \int_{\Pi(\mathrm{M})}\left(\sum_{\phi \in \mathcal{B}_{\mathrm{P}}(\pi)_{\chi}} E(e, \phi) \int_{\mathrm{G}(\mathbb{A})} \boldsymbol{\delta}_{S, D}\left(g^{\prime}\right) H\left(\mathbf{s}, g^{\prime}\right)^{-1} \overline{E\left(g^{\prime}, \phi\right)} \mathrm{d} g^{\prime}\right) \mathrm{d} \pi \text {. }
$$

Theorem 5.2. The function $S^{b}$ admits an analytic continuation to a function which is holomorphic on $\mathcal{T}_{-1 / 2 r}^{D} \cap \mathcal{T}_{-1-1 / 2 r}$, where $r$ is the rank of $\mathrm{G}$.

Proof. By assumption, the height function is invariant under right and left translation by the maximal compact subgroup K. By [19, Corollary 4.1], we have

$$
S^{b}(\mathbf{s})=\sum_{\chi \in \mathcal{X}, \mathrm{P}}^{b} \frac{1}{n(\mathrm{~A})} \int_{\Pi(\mathrm{M})}\left(\sum_{\phi \in \mathcal{B}_{\mathrm{P}}(\pi)_{\chi}} E(e, \phi) \mathcal{J}_{S, D}(\mathbf{s}, E(\phi, \cdot))\right) \mathrm{d} \pi,
$$

where $\mathcal{J}_{S, D}(\mathbf{s}, E(\phi, \cdot))$ is as in Corollary 4.6. Let $K \subset \mathcal{T}_{-1 / 2 r+\epsilon}^{D} \cap \mathcal{T}_{-1-1 / 2 r+\epsilon}$, with $\epsilon>0$, be a compact subset. By Corollary 4.6. for $\mathbf{s} \in K$ and all $k$ the expression

$$
\sum_{\chi \in \mathfrak{X}, \mathrm{P}}^{b} \frac{1}{n(\mathrm{~A})} \int_{\Pi(\mathrm{M})}\left(\sum_{\phi \in \mathcal{B}_{\mathrm{P}}(\pi)_{\chi}}|E(e, \phi)| \cdot\left|\mathcal{J}_{S, D}(\mathbf{s}, E(\phi, \cdot))\right|\right) \mathrm{d} \pi
$$


is bounded by

$$
C(\epsilon, K, k) \sum_{\chi \in \mathfrak{X}, \mathrm{P}}^{b} \frac{1}{n(\mathrm{~A})} \int_{\Pi(\mathrm{M})}\left(\sum_{\phi \in \mathcal{B}_{\mathrm{P}}(\pi)_{\chi}} \boldsymbol{\Lambda}(\phi)^{-k}|E(e, \phi)|^{2}\right) \mathrm{d} \pi,
$$

where $\boldsymbol{\Lambda}(\phi)$ is eigenvalue of the Laplacian $\boldsymbol{\Delta}$ as in Corollary 4.6. The convergence of the last expression follows from [19, Proposition 3.5].

Corollary 5.3. The height zeta function $\mathcal{Z}_{S, D}(\mathbf{s})=\mathcal{Z}_{S, D}(\mathbf{s}, e)$ is holomorphic for $\Re(\mathbf{s}) \in-\left(K_{X}+D\right)+\Lambda_{\mathrm{eff}}(X)^{\circ}$.

\section{The LeAding Pole}

We now establish the analog of Manin's conjecture in the context of integral points, proposed and proved in special cases in [5], [4], [6]. For

$$
\lambda=\sum_{\alpha \in \mathcal{A}} \lambda_{\alpha} \alpha \in \Lambda_{\mathrm{eff}}(X)^{\circ}
$$

the interior of the effective cone of $X$, set

$$
a(\lambda)=\max \left(\max _{\alpha \in \mathcal{A}_{D}} \frac{\kappa_{\alpha}}{\lambda_{\alpha}}, \max _{\alpha \notin \mathcal{A}_{D}} \frac{\kappa_{\alpha}+1}{\lambda_{\alpha}}\right) .
$$

Let

$$
\mathcal{A}(\lambda)=\mathcal{A}(\lambda, D)
$$

be the set of $\alpha$, for which the maximum is achieved and $r(\lambda)=\# \mathcal{A}(\lambda)$ its cardinality. Put

$$
\mathcal{A}_{D}(\lambda)=\mathcal{A}(\lambda) \cap \mathcal{A}_{D}, \quad d(\lambda)=\# \mathcal{A}_{D}(\lambda) .
$$

Theorem 5.2 implies that $\mathcal{Z}_{S, D}(s \lambda)$ has no pole for $\Re(s)>a(\lambda)$ and that possible contributions to the right most poles come from one-dimensional automorphic characters.

Recall that given an automorphic character $\chi$ of $\mathrm{G}(\mathbb{A})$ and an $\alpha \in \Delta(\mathrm{G}, \mathrm{T})$ we can define a Hecke character $\xi_{\alpha}(\chi)$ of $\mathbb{G}_{m}(\mathbb{A})$ by

$$
\xi_{\alpha}(\chi)=\chi \circ \check{\alpha} \text {. }
$$

Then if $\chi=\otimes_{v}^{\prime} \chi_{v}$, we have $\xi_{\alpha}(\chi)=\otimes_{v}^{\prime} \xi_{\alpha, v}\left(\chi_{v}\right)$ with

$$
\xi_{\alpha, v}\left(\chi_{v}\right)=\chi_{v} \circ \check{\alpha} \text {. }
$$

We are only interested in those automorphic $\chi$ which satisfy

$$
\int_{\mathrm{G}(\mathbb{A})} \boldsymbol{\delta}_{S, D}(g) H(s \lambda, g)^{-1} \chi(g) \mathrm{d} g \neq 0
$$

for some $s$ in the domain of absolute convergence. This implies that $\chi$ is right, and in this case also left, invariant under the maximal compact subgroup $\mathrm{K}$ of $\mathrm{G}(\mathbb{A})$, i.e., $\chi \in \mathcal{X}(\mathrm{G})$.

Definition 6.1. Let $\mathcal{X}_{S, D, \lambda}(\mathrm{G}) \subset \mathcal{X}(\mathrm{G})$ be the collection of all characters $\chi=\otimes_{v}^{\prime} \chi_{v}$ such that 
- for all $\alpha \in \mathcal{A}(\lambda) \backslash \mathcal{A}_{D}(\lambda)$ and all $v \notin S$, we have $\xi_{\alpha, v}\left(\chi_{v}\right) \equiv 1$;

- for all $\alpha \in \mathcal{A}_{D}(\lambda)$, and $v \in S$, we have $\xi_{\alpha, v}\left(\chi_{v}\right) \equiv 1$.

Remark 6.2. If $D=\emptyset$ and $\lambda=-K_{X}$ then $\mathcal{X}_{S, D, \lambda}(\mathrm{G})=1$, the trivial character, for any $S$ [19, Proposition 8.6]. If $D=\cup_{\alpha \in \mathcal{A}}$ is the whole boundary and $S=S_{\infty}$ then $\mathcal{X}_{S, D, \lambda}(\mathrm{G})$ is dual to the class group of G, i.e., the quotient

$$
\operatorname{cl}(\mathrm{G}):=\mathrm{G}(\mathbb{A}) / \mathrm{G}(F) \mathrm{K} \prod_{v \mid \infty} \mathrm{G}\left(F_{v}\right) .
$$

This class group is trivial for groups of type $E_{8}, F_{4}$, and $G_{2}$, as the adjoint groups are also simply-connected, and these have class number 1 .

Example 6.3. If $\chi$ is an unramified character of a split semi-simple group over a number field of class number one, then $\chi=1$; this follows from [11, Lemma 4.7], and Corollary 2 on page 486 of [17].

This may fail when the class number of $F$ is not equal to one. E.g., let $F$ be a field with class number two, and let $E$ be the Hilbert class field of $F$. Then $E / F$ is an unramified quadratic extension. Let $\omega_{E / F}$ be the corresponding quadratic character of $\mathbb{A}_{F}^{\times}$. Consider the automorphic character of $\operatorname{PGL}(2)$ given by $\omega_{E / F} \circ$ det. Then this automorphic character is unramified and trivial at the archimedean places. Such characters will contribute to the leading pole of $\mathcal{Z}_{S, D}$.

We have shown that

$$
\mathcal{Z}_{S, D}(s \lambda)=\sum_{\chi \in \mathcal{X}(\mathrm{G})} \int_{\mathrm{G}(\mathbb{A})} \boldsymbol{\delta}_{S, D}(g) H(s \lambda, g)^{-1} \chi(g) \mathrm{d} g+f(s),
$$

with $f$ holomorphic for $\Re(s)>a(\lambda)-\delta$, for some $\delta>0$. Theorem 3.2 combined with basic properties of Hecke $L$-functions shows that for $\chi \in$ $\mathcal{X}(\mathrm{G})$ the integral

$$
\int_{\mathrm{G}(\mathbb{A})} \boldsymbol{\delta}_{S, D}(g) H_{S}(s \lambda, g)^{-1} \chi(g) \mathrm{d} g
$$

admits a regularization of the shape

$$
\prod_{\alpha \in \mathcal{A}(\lambda) \backslash \mathcal{A}_{D}(\lambda)} L_{S}\left(s \lambda_{\alpha}-\kappa_{\alpha}, \xi_{\alpha}(\chi)\right) \cdot h_{\chi}(s) \cdot \prod_{v \in S} \prod_{\alpha \in \mathcal{A}_{D}(\lambda)} L_{v}\left(s \lambda_{\alpha}-\kappa_{\alpha}, \xi_{\alpha, v}\left(\chi_{v}\right)\right) \cdot h_{\chi, v}(s),
$$

with $h_{\chi}$ and $h_{\chi, v}$ holomorphic for $\Re(s)>a(\lambda)-\delta$, for some $\delta>0$. It follows that only $\chi \in \mathcal{X}_{S, D, \lambda}(\mathrm{G})$ contribute to the leading term at $s=a(\lambda)$. By Poisson summation formula, we can rewrite this contribution as

where

$$
\left|\mathcal{X}_{S, D, \lambda}(\mathrm{G})\right| \cdot \int_{\mathrm{G}(\mathbb{A})^{\mathrm{Ker}_{\lambda}}} \boldsymbol{\delta}_{S, D}(g) H(s \lambda, g)^{-1} \mathrm{~d} g
$$

$$
\mathrm{G}(\mathbb{A})^{\operatorname{Ker}_{\lambda}}:=\cap_{\chi \in \mathcal{X}_{S, D, \lambda}(\mathrm{G})} \operatorname{Ker}(\chi)
$$


is the intersection of the kernels of automorphic characters.

Theorem 6.4. The number of $(S, D)$-integral point of bounded height with respect to $\lambda$ is asymptotic to

$$
c \cdot B^{a(\lambda)} \log (B)^{b(\lambda)-1}(1+o(1)), \quad B \rightarrow \infty,
$$

where

$$
b(\lambda)=r(\lambda)-d(\lambda)+\sum_{v \in S} d(\lambda)
$$

and

$$
c=\frac{1}{a(\lambda)(b(\lambda)-1) !} \cdot\left|\mathcal{X}_{S, D, \lambda}(\mathrm{G})\right| \cdot \int_{\mathrm{G}(\mathbb{A})^{\mathrm{Ker}_{\lambda}}} \boldsymbol{\delta}_{S, D}(g) H(s \lambda, g)^{-1} \mathrm{~d} g>0 .
$$

Proof. We adopt the proof in [19, Theorem 9.2], combining it with a Tauberian theorem as in [5, Theorem A.15]. It suffices to establish that the limit

$$
\lim _{s \rightarrow a(\lambda)}(s-a(\lambda))^{b(\lambda)} \int_{\mathrm{G}(\mathbb{A})^{\operatorname{Ker}_{\lambda}}} \boldsymbol{\delta}_{S, D}(g) H(s \lambda, g)^{-1} \mathrm{~d} g>0 .
$$

Note that there exists a finite set of $\gamma_{j} \in \mathrm{G}(F)$ such that

$$
\mathrm{G}(\mathbb{A})=\sqcup \gamma_{j} \mathrm{G}(\mathbb{A})^{\mathrm{Ker}_{\lambda}}
$$

and that the local and global integrals over each of these cosets are comparable, upto a constant. In particular, it suffices to establish that

$$
\lim _{s \rightarrow a(\lambda)}(s-a(\lambda))^{b(\lambda)} \int_{\mathrm{G}(\mathbb{A})} \boldsymbol{\delta}_{S, D}(g) H(s \lambda, g)^{-1} \mathrm{~d} g>0,
$$

which follows from Theorem 3.2 and the definitions.

We now specialize to the case when $\lambda=-\left(K_{X}+D\right)$, the log-anticanonical line bundle. The first condition in Definition 6.1 implies that if $\chi \in \mathcal{X}_{S, D, \lambda}(\mathrm{G})$ and $\alpha \notin \mathcal{A}_{D}$ then $\xi_{\alpha, v}\left(\chi_{v}\right) \equiv 1$ for all $v$. Combining with the second condition, we see that $\xi_{\alpha, v}\left(\chi_{v}\right) \equiv 1$ for all $\alpha \in \mathcal{A}$ and all $v \in S$. In particular, for $\chi \in \mathcal{X}_{S, D, \lambda}(\mathrm{G})$ the integrals

$$
\int_{\mathrm{G}(\mathbb{A})} \boldsymbol{\delta}_{S, D}(g) H(s \lambda, g)^{-1} \chi(g) \mathrm{d} g
$$

do not depend on $\chi$ and equal

$$
\int_{\mathrm{G}\left(\mathbb{A}_{S}\right)} \boldsymbol{\delta}_{S, D}(g) H(s \lambda, g)^{-1} \mathrm{~d} g \cdot \prod_{v \in S} \int_{\mathrm{G}\left(F_{v}\right)} H_{v}\left(s \lambda, g_{v}\right)^{-1} \mathrm{~d} g_{v}
$$

We can now describe the constant $c$ appearing in (19) in terms of Tamagawatype constants. First, we recall some notation. By [8], the boundary strata of $X \backslash \mathrm{G}$ are in bijection with subsets $A \subset \mathcal{A}$, i.e., there is a unique stratum

$$
Z_{A}:=\cap_{\alpha \in A} D_{\alpha} .
$$

For split $\mathrm{G}$, each such stratum $Z_{A}$ contains $F_{v}$-adic points.

In the terminology of [5, Section 3], at each place $v$, the analytic Clemens polytope $\mathcal{C}_{v}^{\text {an }}(D)$ of $D$ has a unique face of maximal dimension, it corresponds 
to $Z_{\mathcal{A}_{D}}\left(F_{v}\right)$. Put $d:=\operatorname{dim}\left(\mathcal{C}_{v}^{\text {an }}(D)\right)+1$; we have $d=\# \mathcal{A}_{D}$, the codimension of the stratum $Z_{\mathcal{A}_{D}}$ (see also [5, Section 5.3.2]). In this situation, for each $v \in S$, there is a distinguished $v$-adic measure $\tau_{v}^{\max }$ on $D\left(F_{v}\right)$ considered in [5, Section 4]. It is supported on $Z_{\mathcal{A}_{D}}\left(F_{v}\right)$ and the corresponding volumes are given by

$$
\tau_{v}^{\max }\left(D\left(F_{v}\right)\right)=\prod_{\alpha \in \mathcal{A}_{D}} \frac{1}{\kappa_{\alpha}} \cdot \lim _{s \rightarrow 1}(s-1)^{d} \int_{\mathrm{G}\left(F_{v}\right)} H_{v}\left(s \lambda, g_{v}\right)^{-1} \mathrm{~d} g_{v} .
$$

Furthermore, there is an adelic measure on the integral adeles on $U:=X \backslash D$,

which in our case takes the form:

$$
\tau_{(X, D)}^{S}\left(U\left(\mathbb{A}_{S}\right)^{\mathrm{int}}\right)=\prod_{\alpha \notin \mathcal{A}_{D}} \frac{1}{\kappa_{\alpha}+1} \cdot \lim _{s \rightarrow 1}(s-1)^{r-d} \int_{\mathrm{G}\left(\mathbb{A}_{S}\right)} \boldsymbol{\delta}_{S}(g) H_{S}\left(s \lambda, g_{v}\right)^{-1} \mathrm{~d} g .
$$

It follows that

$$
c=\frac{1}{(b-1) !} \cdot\left|\mathcal{X}_{S, D, \lambda}(\mathrm{G})\right| \cdot \tau_{(X, D)}^{S}\left(U\left(\mathbb{A}_{S}\right)^{\mathrm{int}}\right) \cdot \prod_{v \in S} \tau_{v}^{\max }\left(D\left(F_{v}\right)\right),
$$

where

$$
b:=(r-d)+\sum_{v \in S} d
$$

Formula (20) interpolates between Peyre's Tamagawa-type constant for leading terms in asymptotics of rational points and the "concentration of counting measures to the Satake boundary" for asymptotics for integral points on G, established in [13].

\section{REFERENCES}

1. J. Arthur, A trace formula for reductive groups. I. Terms associated to classes in $G(\mathbf{Q})$, Duke Math. J. 45 (1978), no. 4, 911-952. MR MR518111 (80d:10043)

2. Y. Benoist and H. Oh, Effective equidistribution of $S$-integral points on symmetric varieties, 2007, arXiv:0706.1621.

3. M. Borovoi and Z. Rudnick, Hardy-Littlewood varieties and semisimple groups, Invent. Math. 119 (1995), no. 1, 37-66. MR 1309971 (96b:11084)

4. A. Chambert-Loir and Y. Tschinkel, Integral points of bounded height on partial equivariant compactifications of vector groups, 2009, arXiv:0912.4751.

5. _ Igusa integrals and volume asymptotics in analytic and adelic geometry, Confluentes Mathematici 2 (2010), no. 3, 351-429.

6. Integral points of bounded height on toric varieties, 2010, preprint.

7. C. De Concini and C. Procesi, Complete symmetric varieties, Invariant theory (Montecatini, 1982), Lecture Notes in Math., vol. 996, Springer, Berlin, 1983, pp. 1-44. MR 718125 (85e:14070)

8. _ Complete symmetric varieties, Invariant theory (Montecatini, 1982), Lecture Notes in Math., vol. 996, Springer, Berlin, 1983, pp. 1-44. MR 85e:14070

9. W. Duke, Z. Rudnick, and P. Sarnak, Density of integer points on affine homogeneous varieties, Duke Math. J. 71 (1993), no. 1, 143-179. MR 94k:11072

10. A. Eskin and C. McMullen, Mixing, counting, and equidistribution in Lie groups, Duke Math. J. 71 (1993), no. 1, 181-209. MR 1230290 (95b:22025) 
11. A. Gorodnik, F. Maucourant, and H. Oh, Manin's and Peyre's conjectures on rational points and adelic mixing, Ann. Sci. Éc. Norm. Supér. (4) 41 (2008), no. 3, 383-435. MR 2482443 (2010a:14047)

12. A. Gorodnik and A. Nevo, Counting lattice points, 2009, arXiv:0903.1515.

13. A. Gorodnik, H. Oh, and N. Shah, Integral points on symmetric varieties and Satake compactifications, Amer. J. Math. 131 (2009), no. 1, 1-57. MR 2488484 (2009m:22017)

14. B. Hassett and Y. Tschinkel, Density of integral points on algebraic varieties, Rational points on algebraic varieties, Progr. Math., vol. 199, Birkhäuser, Basel, 2001, pp. 169197. MR 1875174 (2003a:14034)

15. M. Morishita and T. Watanabe, On S-Hardy-Littlewood homogeneous spaces, Internat. J. Math. 9 (1998), no. 6, 723-757. MR 1644303 (99g:11082)

16. H. Oh, Uniform pointwise bounds for matrix coefficients of unitary representations and applications to Kazhdan constants, Duke Math. J. 113 (2002), no. 1, 133-192. MR MR1905394 (2003d:22015)

17. V. Platonov and A. Rapinchuk, Algebraic groups and number theory, Pure and Applied Mathematics, vol. 139, Academic Press Inc., Boston, MA, 1994. MR MR1278263 (95b:11039)

18. J. Shalika, R. Takloo-Bighash, and Y. Tschinkel, Rational points on compactifications of semi-simple groups of rank 1, Arithmetic of higher-dimensional algebraic varieties (Palo Alto, CA, 2002), Progr. Math., vol. 226, Birkhäuser Boston, Boston, MA, 2004, pp. 205-233. MR MR2029871 (2004m:11104)

19. , Rational points on compactifications of semi-simple groups, J. Amer. Math. Soc. 20 (2007), no. 4, 1135-1186 (electronic). MR 2328719 (2008g:14034)

Department of Mathematics, UiC, 851 S. Morgan str., Chicago, IL 60607, U.S.A.

E-mail address: rtakloo@math.uic.edu

Courant Institute of Mathematical Sciences, N.Y.U., 251 Mercer str., New YORK, NY 10012, U.S.A.

E-mail address: tschinkel@cims.nyu.edu 